\title{
Review of: "Orthogonal Gene Engineering Enables CD8+ T Cells to Control Tumors through a Novel PD-1+TOX- indifferent Synthetic Effector State"
}

\author{
Gang Zhou ${ }^{1}$ \\ 1 Augusta University
}

Potential competing interests: The author(s) declared that no potential competing interests exist.

In this study Corria-Osorio et al report that tumor-specific CD8 T cells orthogonally engineered to express an IL-2Rbg binding IL-2 variant (IL2v), PD1-decoy (Pd1d) and IL-33 acquired an unique effector state and exhibited potent antitumor activity after adoptive transfer into tumor-bearing hosts. The authors show that IL2v and IL33 synthetically drove the development of PD-1+TOXlow/negGzmC+ donor T cells, with IL2v promoting CD8 T cell stemness, persistence, downregulation of TOX while IL33 endowing effector polyfunctionality. Strikingly, ACT with Pd1dIL2vIL33 CD8 T cells led to transcriptional reprogramming of the endogenous CD8 T cells which contributed to the antitumor effect. The findings illustrate how rationally designed $\mathrm{T}$ cell engineering can fundamentally improve $\mathrm{T}$ cell fitness and function in the tumor microenvironment. The ability of the genetically modified CD8 T cells to expand, differentiate and persist without the need of host lymphodepletion is remarkable and clearly has translational significance. The authors should be congratulated for this thoughtful and elegantly performed important work! It would be great if the authors can elaborate more on the following points to help readers better understand the model system and appreciate the potential application of the findings.

1. It is not clear why donor OT1 T cells were transferred twice (day0 and day3). What would happen if donor T cells were transferred once with T cells in a number equal to the sum of two transfers? In the case of two transfers, there is the concern that the early transferred T cells may have an advantage in terms of expansion/differentiation and may inhibit the expansion of later transferred T cells.

1. The authors showed that PD1 (and Tim3) did not play a role in T cell phenotype and function in the model. It is curious why the $T$ cell engineering always included Pd1d throughout the study? For adoptive transfer of $P d 1 d / 2 v / 33$, the donor cells were a 1:1 mix of Pd1d/2v-transduced T cells and Pd1d/33-transduced T cells. What would happen if the donor $T$ cells are engineered to simultaneously express Pd1d/2v/33 (or only $2 \mathrm{v} / 33$ ) through a single viral vector? This may address the issue of IL33 attrition which contributed to tumor escape. This may also shed light on autocrine vs paracrine effect of the engineered T cells.

1. Published studies indicate that $\mathrm{T}$ cells in different activation (naïve, effector, memory and exhaustion) state are associated with unique epigenetic landscape. scRNAseq coupled with ATACseq might be more informative to define 
the unique novel synthetic effector state described in this study. This may also provide insight into why PD1/Tim3 inhibitory receptors are inconsequential to the cluster $5 \mathrm{~T}$ cells.

1. The study suggested the activation of the endogenous CD8 T cells. Is there evidence of antigen epitope spreading? Were the endogenous CD8 T cells reactive to wildtype B16 cells in vitro? Were the endogenous CD8 T cells reactive to OVA or other tumor-associated antigens? Was there evidence of DC recruitment and activation in the tumor since the authors showed that draining lymph nodes were not required to T cell activation?

1. This study exclusively used the OT1/OVA-expressing tumor models. It would further strengthen the translational potential if a model featuring authentic tumor antigen, such as the pmel1/gp100 system, can be used to validate the major findings. 